\title{
Prediction of Fatigue Life for a New 2-DOF Compliant Mechanism by Clustering-Based ANFIS Approach
}

\author{
Ngoc Thoai Tran, ${ }^{1}$ Thanh-Phong Dao $\mathbb{D}^{2,3}$ Thao Nguyen-Trang $\mathbb{D}^{4,5}$ and Che-Ngoc Ha $\mathbb{D}^{5}$ \\ ${ }^{1}$ Faculty of Mechanical Engineering, Industrial University of Ho Chi Minh City, Ho Chi Minh City, Vietnam \\ ${ }^{2}$ Division of Computational Mechatronics, Institute for Computational Science, Ton Duc Thang University, \\ Ho Chi Minh City, Vietnam \\ ${ }^{3}$ Faculty of Electrical \& Electronics Engineering, Ton Duc Thang University, Ho Chi Minh City, Vietnam \\ ${ }^{4}$ Division of Computational Mathematics and Engineering, Institute for Computational Science, Ton Duc Thang University, \\ Ho Chi Minh City, Vietnam \\ ${ }^{5}$ Faculty of Mathematics and Statistics, Ton Duc Thang University, Ho Chi Minh City, Vietnam
}

Correspondence should be addressed to Che-Ngoc Ha; chengocha@tdtu.edu.vn

Received 1 November 2020; Revised 27 January 2021; Accepted 23 February 2021; Published 2 March 2021

Academic Editor: Mingwei Lin

Copyright (c) 2021 Ngoc Thoai Tran et al. This is an open access article distributed under the Creative Commons Attribution License, which permits unrestricted use, distribution, and reproduction in any medium, provided the original work is properly cited.

\begin{abstract}
Two-degree-of-freedom (2-DOF) compliant mechanism has some outstanding characteristics in accurate positioning systems. Studying the fatigue life for the 2-DOF compliant mechanism is a meaningful task to ensure a long working. However, a study for fatigue life prediction of this mechanism has not been conducted so far. In this article, a method for fatigue life prediction of 2DOF compliant mechanism is developed for the first time. This method is the combining of the differential evolution algorithm and the adaptive neuro-fuzzy inference system (ANFIS) with subtractive clustering. The numerical results on two case studies consisting of material steel A-36 and the material AL 6061-T6 show that the accuracy of the proposed method is very high. Compared to the actual fatigue life, the root mean square error of the proposed method lies in the range [1.7, 3.97] cycles for Case 1 and $[2.03,10.38]$ cycles for Case 2 . The statistical test also indicates that the proposed method outperforms the traditional method using triangular membership function, bell-shape, and Gaussian membership function, with the significance level from 0.05 to 0.1 . These results demonstrate the feasibility of the proposed approach in fatigue life prediction of 2-DOF compliant mechanism.
\end{abstract}

\section{Introduction}

Two-degree-of-freedom (2-DOF) compliant mechanism is a monolithic structure that has been widely utilized in ultrahigh precise engineering. Basically, the 2-DOF compliant mechanism inherits outstanding characteristics such as minimal positioning error, free friction, reduced assemble, and easy machining [1]. Several applications of this mechanism can be found in the nanopositioning system [2], micropositioning system [3-9], space pointing mechanism [10], and so on. Although the 2-DOF compliant mechanism proposes excellent advantages in precision engineering and manipulators, this mechanism still exists major drawbacks, including nonlinear kinematic behaviors and fatigue influences. Considering the first disadvantage, nonlinear kinematic behaviors can be modeled and analyzed by pseudorigid-body model [11], compliance matrix method [12], and pseudostatic model [13]. Regarding the second disadvantage, fatigue influences on the 2-DOF compliant mechanism can result in a failure under dynamic working conditions.

In similarity to rigid-link mechanisms, fatigue life of the 2-DOF complaint mechanism is a term indicating how long an object will last before it fails due to concentrated stresses. Evaluation of the fatigue life is a critically important task in design process of compliant mechanisms so as to guarantee the working safety and reliability. Practically, compliant mechanisms are often subjected to cyclic and reversed loads. 
Under varying stress cycles, fatigue phenomena can appear in compliant mechanisms. So, it is necessary to predict the fatigue life to avoid any damage. Nevertheless, modeling of the fatigue life for the 2-DOF compliant mechanism is so complicated task because the 2-DOF compliant mechanism is a complex structure that has an integration of kinematic behaviors and mechanical ones. Therefore, the problem of fatigue prediction has been interested to research recently to prevent undesired failures. In [14], the artificial neural networks (ANNs) were used to predict the fatigue life of polyamide- 6 polymer. The results showed that the ANNbased approach can achieve a better performance than conventional methods. The adaptive neuro-fuzzy inference system (ANFIS) was used to model the fatigue behavior of a multidirectional laminate under four different loading conditions [15]. In the above study, both grid partitioning and subtractive clustering were used to build the ANFIS models. Nevertheless, a method for setting subtractive clustering parameters has not been conducted. The research in [16] provided a review of fatigue life modeling, fatigue life prediction, and application to composite structure design. The research studies in $[17,18]$ proposed a method for solving the partial differential equations using the deep neural network (DNN). This approach was then utilized for many applications in the field of Computational Mechanics. Various soft computing methods for predicting the fatigue life of different material systems were also studied in [19-28]. However, it can be noted that a study predicting the fatigue life for 2-DOF compliant mechanism has not been conducted so far.

To fulfill the mentioned research gap, this paper develops a method based on the adaptive neuro-fuzzy inference system (ANFIS) with subtractive clustering for fatigue life prediction of 2-DOF compliant mechanism. Furthermore, for searching the optimal radius parameters of the clustering algorithm, the 10-fold cross-validation and the differential evolution (DE) algorithm are used. Particularly, the average mean square error of 10 -folds is evaluated and optimized using the DE. The proposed framework is applied to predict the fatigue life of two cases of 2-DOF compliant mechanism consisting of the material steel A-36 and the material AL 6061-T6 based on their design variables.

The rest of the paper is organized as follows. The related works including the adaptive neuro-fuzzy inference system, the subtractive clustering, and the differential evolution algorithm are presented in Section 2. The proposed framework is presented in Section 3. The new method is applied to predict fatigue life for 2-DOF compliant mechanism consisting of steel materials A-36 and AL 6061-T6. In the conclusion, the main contribution, the results, and a few further research directions are summarized and discussed.

\section{Related Works}

2.1. Fuzzy Set. Fuzzy set, which was introduced by La [29], is a well-known theory for modeling data uncertainty. A fuzzy set $A$ on universe $X$ is a set defined by the membership function $\mu_{A}(x)$ which is a mapping from $X$ into the interval $[0,1]$. If $\mu_{A}(x)=1, x$ completely belongs to the fuzzy set $A$. If
$\mu_{A}(x)=0, x$ does not belong to the fuzzy set. If $0<\mu_{A}(x)<1, x$ is a partial member of the fuzzy set $A$. There are several ways to define the membership function $\mu_{A}(x)$. Some well-known membership functions are the trapezoidal membership function, the triangular membership function, and the Gaussian membership function.

2.2. Fuzzy Inference System. The fuzzy inference system (FIS) [30] is a system that uses fuzzy set theory to map inputs to output (independent variables to dependent variable in the case of regression). There are two main types of FIS including the Mamdani and the Sugeno systems. This paper concentrates on the Sugeno system which builds a separate subregression model corresponding to each rule and calculates the final output as the weight-mean of all subregression outputs. For example, let $X$ be a script variable that can be mapped to two corresponding fuzzy sets $A_{1}$ and $A_{2}, Y$ be a script variable that can be mapped to two corresponding fuzzy sets $B_{1}$ and $B_{2}$, and $Z$ be the output or dependent variable. $Z$ can be computed via the Sugeno fuzzy logic inference as follows:

If $X$ is $A_{1}$ and $Y$ is $B_{1}$, then $z_{1}=p_{1} x+q_{1} y+r_{1}$ with the degree of applicability is $\lambda_{1}$

If $X$ is $A_{2}$ and $Y$ is $B_{2}$, then $z_{2}=p_{2} x+q_{2} y+r_{2}$ with the degree of applicability is $\lambda_{2}$

If $X$ is $A_{1}$ and $Y$ is $B_{2}$, then $z_{3}=p_{3} x+q_{3} y+r_{3}$ with the degree of applicability is $\lambda_{3}$

Finally, the output $Z$ is computed using the formula $z=\lambda_{1} z_{1}+\lambda_{2} z_{2}+\lambda_{3} z_{3} / \lambda_{1}+\lambda_{2}+\lambda_{3}$

In the above formulas, $z_{i}$ is the component predicted value of fuzzy rule $i$, the predicted value $z$ can be considered as the weight-mean of all component predicted values $z_{i}$, and $p_{i}, q_{i}, r_{i}$ are user-defined. A disadvantage of the Sugeno FIS is that expert knowledge is required to determine the fuzzy set parameters and the coefficients $p, q$, and $r$; therefore, the FIS is a nonoptimal model and lacks learning ability. To improve the performance of FIS, [31] presented a hybrid model called ANFIS that combines the Sugeno FIS and the artificial neural networks. ANFIS can take advantage of the fuzzy reasoning of FIS and the learning capability of ANN. A brief review of ANFIS is presented as follows.

2.3. Adaptive Neuro-Fuzzy Inference System. Soft computing refers to an approach that attempts to model the behavior of a complex system so that an approximate solution of this system can be provided. By the approximation, soft computing not only can provide a relatively good result but also can significantly reduce the computational cost. Therefore, this approach has many applications in engineering problems, such as civil engineering [32-34], mining engineering [35-37], and agricultural and biological engineering [38]. The adaptive neuro-fuzzy inference system (ANFIS) proposed by [31] can take advantage of the fuzzy reasoning of FIS and the learning capability of ANN. Therefore, ANFIS has been successfully performed in classification and prediction tasks and has become one of the most well-known 
soft computing methods so far. A simple illustration of an ANFIS model is presented in Figure 1.

It can be seen from Figure 1 that ANFIS consists of five layers that perform different functions. Let $O_{l i}$ be the output of the $i$ th node of the $l$ th layer. Assuming that the variable $X$ can be partitioned into two fuzzy sets, $A_{1}$ and $A_{2}$, whereas the variable $Y$ can be partitioned into two fuzzy sets $B_{1}$ and $B_{2}$. In Layer 1 , each node calculates the membership function $\mu_{A_{j}}(x)$ and $\mu_{B_{i}}(y), j=1,2$. In other words, $O_{1 i}=\mu_{A_{j}}(x)$ for $i=1,2$ and $O_{1 i}=\mu_{B_{i}}(y)$ for $i=3,4$. Some well-known membership functions are, namely, Gaussian membership function $\mu_{A}(x)=\exp \left(-(x-c)^{2} / 2 \sigma^{2}\right)$, triangular membership function $\mu_{A}(x)=\max (\min ((x-1 / b-a),(c-x /$ $c-b)), 0)$, and generalized bell membership function $\mu_{A}(x)=1 /\left(1+|x-c / a|^{2 b}\right)$, where $a, b$, and $c$ are called as premise parameters. Each node in Layer 2 is the AND operator that calculates the joint membership degree of $(x, y)$ in $A_{j}$ and $B_{k}, j, k=1,2$. Finding the product of individual membership degrees is a popular method modeling the AND operator; therefore, $O_{2 i}=T_{P}\left(\mu_{A_{j}}(x), \mu_{B_{k}}\right.$ $(x))=\mu_{A_{j}}(x) \mu_{B_{k}}(x), j, k=1,2$. Another operator for calculating the joint membership degree is defined as $T_{M}\left(\mu_{A_{j}}(x), \mu_{B_{k}}(x)\right)=\min \left\{\mu_{A_{j}}(x), \mu_{B_{k}}(x)\right\}$. In fact, both $T_{P}$ and $T_{M}$ are some specific examples of a $t$-norm which is used to generalize the conjunction in fuzzy logic. For all $a, b$ in $[0$, 1], $T_{P}(a, b) \leq T_{M}(a, b)$; that is, the logical strength represented by $T_{P}$ is stronger than $T_{M}$. For more details of $t$ norms, readers can refer to [39]. As shown in Figure 1, node 1 in Layer 2 calculates the membership degree of $X$ is $A_{1}$ and $Y$ is $B_{1}$, whereas node 2 in Layer 2 calculates the membership degree of $X$ is $A_{2}$ and $Y$ is $B_{2}$. Certainly, more combinations between $A_{\mathrm{i}}$ and $B_{k}$, for example, $X$ is $A_{1}$ and $Y$ is $B_{2}$, can be handled. For the sake of convenience, only two nodes are illustrated in Figure 1. Let $w_{i}$ be the $i$ th rule membership or firing strength obtained in Layer 2. Layer 3 is utilized for the normalization purpose. In this layer, the output of node $i$ is the ratio of the $i$ th rule's firing strength to the sum of all rules' firing strengths. For example, from ANFIS architecture in Figure 1, it can be implied that $O_{3 i}=\overline{w_{i}}$ $=w_{i} / w_{1}+w_{2}, i=1,2$. Layer 4 calculates the reasoning result for each fuzzy rule using the formula $O_{4 i}=\overline{w_{i}} f_{i}=\overline{w_{i}}\left(p_{i} x+\right.$ $\left.q_{i} y+r_{i}\right)$ where $p_{i}, q_{i}$ and $r_{i}$ are called consequent parameters. Finally, Layer 5 calculates the overall output using the sum of all $O_{4 i}$, that is, $f=O_{51}=\sum_{i=1}^{2} \bar{w}_{i} f_{i}$.

ANFIS can optimize all parameters using a hybrid learning method. In the forward pass, the least-squares method is utilized to identify the consequent parameters in Layer 4 . In the backward pass, the gradient descent method is utilized to identify the premise parameters in Layer 1 . Therefore, the ANFIS inherits both the fuzzy reasoning of FIS and the learning abilities of ANN.

The remaining problem of ANFIS is how to identify the number of fuzzy sets for each variable. The conventional ANFIS uses the grid partitioning method that requires a given number of fuzzy sets defined by expert knowledge. Furthermore, this method causes the dimensional curse when applied to large-scale data. For example, given an input of 12 variables, if each variable

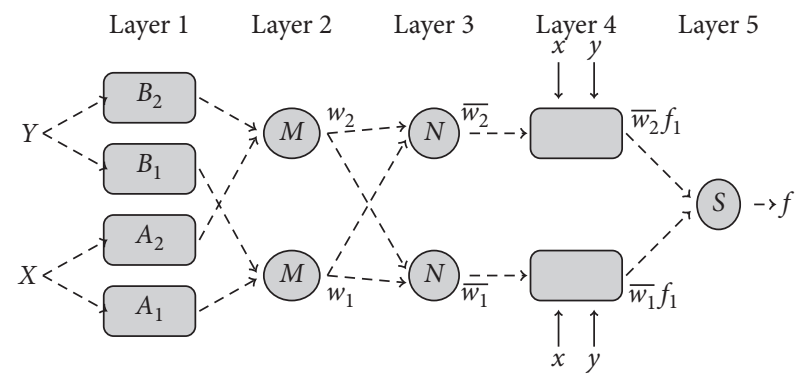

Figure 1: An example of the ANFIS system.

can be partitioned into two fuzzy sets, the grid partitioning method results in $2^{12}=4096$ rules. This number is very large for any practical learning method. Therefore, the conventional ANFIS with the grid partitioning method might be not completely objective and might be infeasible when dealing with large-scale data. An alternative method is using ANFIS with subtractive clustering technique, which is presented in the following section.

2.4. Subtractive Clustering. Cluster analysis is to discover the underlying structure of a dataset by partitioning the data into groups such that each obtained cluster (group) is a set of similar data points [40]. In other words, all the elements in a cluster share the same characteristic behavior of the system. Therefore, each cluster's center can be considered as the basis of a rule that represents a system behavior. Because clustering can create a concise representation of the system behavior, it can reduce the number of fuzzy rules or prevent rule explosion. Let $\left\{\mathbf{x}_{1}, \mathbf{x}_{2}, \ldots, \mathbf{x}_{N}\right\}$ be $N d$-dimensional normalized data points. Subtractive clustering [41], which is often applied to ANFIS, is summarized in the following steps:

Step 1: normalize the data set, set $k=1$, and calculate the likelihood that each data point is a cluster center, using

$$
P_{i}=\sum_{j=1}^{N} \prod_{m=1}^{d} \exp \left(-\frac{4}{r_{m}}\left\|x_{i m}-x_{j m}\right\|^{2}\right),
$$

where $P_{i}$ is the likelihood of point $\mathbf{x}_{i}$ and $r_{m}$ is the neighborhood radius in dimension $m$.

Step 2: select the data point with the highest likelihood as the $k$ th cluster's center.

Step 3: update the likelihood that each data point is a cluster center, using formula

$$
P_{i}:=P_{i}-P_{k}^{*} \prod_{m=1}^{d} \exp \left(-\frac{4}{\eta r_{m}}\left\|x_{i m}-x_{k m}^{*}\right\|^{2}\right)
$$

where $\mathbf{x}_{k}^{*}$ and $P_{k}^{*}$ denote the $k$ th cluster center and its likelihood, respectively. $\eta>1$ is a constant.

Using formula (2), the closer the distance between $\mathbf{x}_{i}$ and $\mathbf{x}_{k}^{*}$ is, the more reduction of its likelihood is. Therefore, all points with an updated $P_{\mathrm{i}}$ of 0 are 
assigned to cluster $k$ and are not considered in the next steps.

Step 4: repeat Steps 2 and 3 until all points are assigned to their clusters.

In the above algorithm, the value of $r_{m}$ has an important effect on the clustering result. When $r_{m}$ tends to zero, the algorithm tends to result in $N$ single clusters, each of which has only one element. In contrast, when $r_{m}$ tends to one, the algorithm tends to result in a single cluster of $N$ elements. It is not easy to determine a suitable value for $r_{m}$ that can work well for all case studies. Therefore, an additional framework for optimizing the set of $r_{m}$ is developed. The developed framework is based on the differential evolution algorithm, which will be presented in the next section.

2.5. Differential Evolution Algorithm. The differential evolution algorithm, DE, which is a well-known global search method based on population, is designed to solve both continuous and discrete problems [42]. To clarify the used notation, this article refers to the minimization of the objective function $f(\mathbf{x})$, where $\mathbf{x}$ is a vector of $d$ variables in the decision space $S=\left[\mathbf{x}_{l}, \mathbf{x}_{u}\right]$ and $\mathbf{x}_{l}$ and $\mathbf{x}_{u}$ are the vectors of lower bound and upper bound, respectively. The DE seeks an optimal solution through generations (iterations). In each generation, the DE evolves a population $P$ of size $N P$, $P=\left\{\mathbf{x}_{1}, \mathbf{x}_{2}, \ldots, \mathbf{x}_{\mathrm{NP}}\right\}$. Each element in this set or each feasible solution $\mathbf{x}_{i}, i=\overline{1, \mathrm{NP}}$ is called a chromosome, which is a vector of $d$ variables, so-called genes. The four major operators of the DE algorithm, which include initialization, mutation, crossover, and selection, are briefly summarized as follows.

2.5.1. Initialization. In this phase, an initial population of $N P$ chromosomes is generated through a random sampling technique. Specifically, each individual is represented as a chromosome containing $N$ genes and is generated by

$$
x_{i, j}=x_{j}^{l}+r_{0} \times\left(x_{j}^{u}-x_{j}^{l}\right) \quad i=1,2, \ldots, \mathrm{NP} ; j=1,2, \ldots, d \text {, }
$$

where $R_{0} \sim \mathrm{U}(0,1), N P$ is the size of the population, and $x_{j}^{l}$ and $x_{j}^{u}$ are the lower and upper limits of $x_{j}$, respectively [43].

2.5.2. Mutation. In this phase, each individual $\mathbf{x}_{i}$ in the current population generates a mutant vector using some mutation operators listed as follows:

$$
\begin{aligned}
& \text { rand/1: } \mathbf{v}_{i}=\mathbf{x}_{r_{1}}+F \times\left(\mathbf{x}_{r_{2}}-\mathbf{x}_{r_{3}}\right) \\
& \operatorname{rand} / 2: \mathbf{v}_{i}=\mathbf{x}_{r_{1}}+F \times\left(\mathbf{x}_{r_{2}}-\mathbf{x}_{r_{3}}\right)+F \times\left(\mathbf{x}_{r_{4}}-\mathbf{x}_{r_{5}}\right) \\
& \text { best/1: } \mathbf{v}_{i}=\mathbf{x}^{*}+F \times\left(\mathbf{x}_{r_{1}}-\mathbf{x}_{r_{2}}\right) \\
& \text { best/2: } \mathbf{v}_{i}=\mathbf{x}^{*}+F \times\left(\mathbf{x}_{r_{1}}-\mathbf{x}_{r_{2}}\right)+F \times\left(\mathbf{x}_{r_{3}}-\mathbf{x}_{r_{4}}\right) \\
& \text { current-to-best/1: } \quad \mathbf{v}_{i}=\mathbf{x}_{i}+F \times\left(\mathbf{x}^{*}-\mathbf{x}_{i}\right)+F \times\left(\mathbf{x}_{r_{1}}-\right. \\
& \left.\mathbf{x}_{r_{2}}\right)
\end{aligned}
$$

where $r_{1}, r_{2}, r_{3}, r_{4}$, and $r_{5}$ are mutually exclusive integers randomly chosen in $\{1,2, \ldots, N P\}, F$ is the scale factor randomly selected in the range $[0,2]$, and $\mathbf{x}^{*}$ is the best individual in the current population.

2.5.3. Crossover. After completing mutation, each target vector $\mathbf{x}_{i}$ produces a trial vector $\mathbf{u}_{i}$ by substituting some components of the vector $\mathbf{x}_{i}$ by some components of the mutant vector $\mathbf{v}_{i}$ through the following binomial crossover operation [43]:

$$
u_{i j}= \begin{cases}v_{i j} & \text { if rand }[0,1] \leq \mathrm{CR} \\ x_{i j} & \text { otherwise }\end{cases}
$$

where $i \in\{1,2, \ldots, N P\}, j \in\{1,2, \ldots, d\}$, and $C R$ is the crossover control parameter chosen in $[0,1]$.

2.5.4. Selection. In this phase, each trial vector $\mathbf{u}_{i}$ created after the crossover phase will be evaluated and compared with the target vector $\mathbf{x}_{i}$ to choose a better individual for the next generation.

2.6. Evaluating an ANFIS Model. Let $y_{i}$ and $\hat{y}_{i}$ be the $i$ th actual and predictive data points, respectively. Some criteria for evaluating the ANFIS model are presented as follows:

Mean absolute error:

$$
\operatorname{MAE}=\frac{1}{N} \sum_{i=1}^{N}\left|y_{i}-\widehat{y}_{i}\right| .
$$

Mean squared error:

$$
\operatorname{MSE}=\frac{1}{N} \sum_{i=1}^{N}\left(y_{i}-\widehat{y}_{i}\right)^{2} .
$$

Mean absolute percentage error:

$$
\text { MAPE }=\frac{1}{N} \sum_{i=1}^{N}\left(\frac{\left|y_{i}-\hat{y}_{i}\right|}{y_{i}} .100\right) \% .
$$

2.7. K-Fold Cross-Validation Method. K-fold cross-validation is a typical process being often incorporated into the training process for avoiding the over-fitting problem [44-46]. In this procedure, the original training data set is randomly divided into $k$ subsets. $k-1$ subsets are used as new training data sets to develop an ANFIS model. The remainder is used for evaluating the model using the criteria presented above. This process is repeated $k$ times until every subset is used once for evaluating. Finally, the overall performance is calculated by taking the average of $k$ individual measures. The ANFIS with the lowest average of MAE, MSE, and MAPE can be considered as the most suitable model. Additionally, it can be noted that the test data set is not used in the training and cross-validation process but is only used in the final validation when the optimized ANFIS has been already built. An illustration for $k$-fold cross-validation process is presented in Figure 2 where $k$ is set as three and $\alpha$ is the ratio of the test set compared to the data set. 


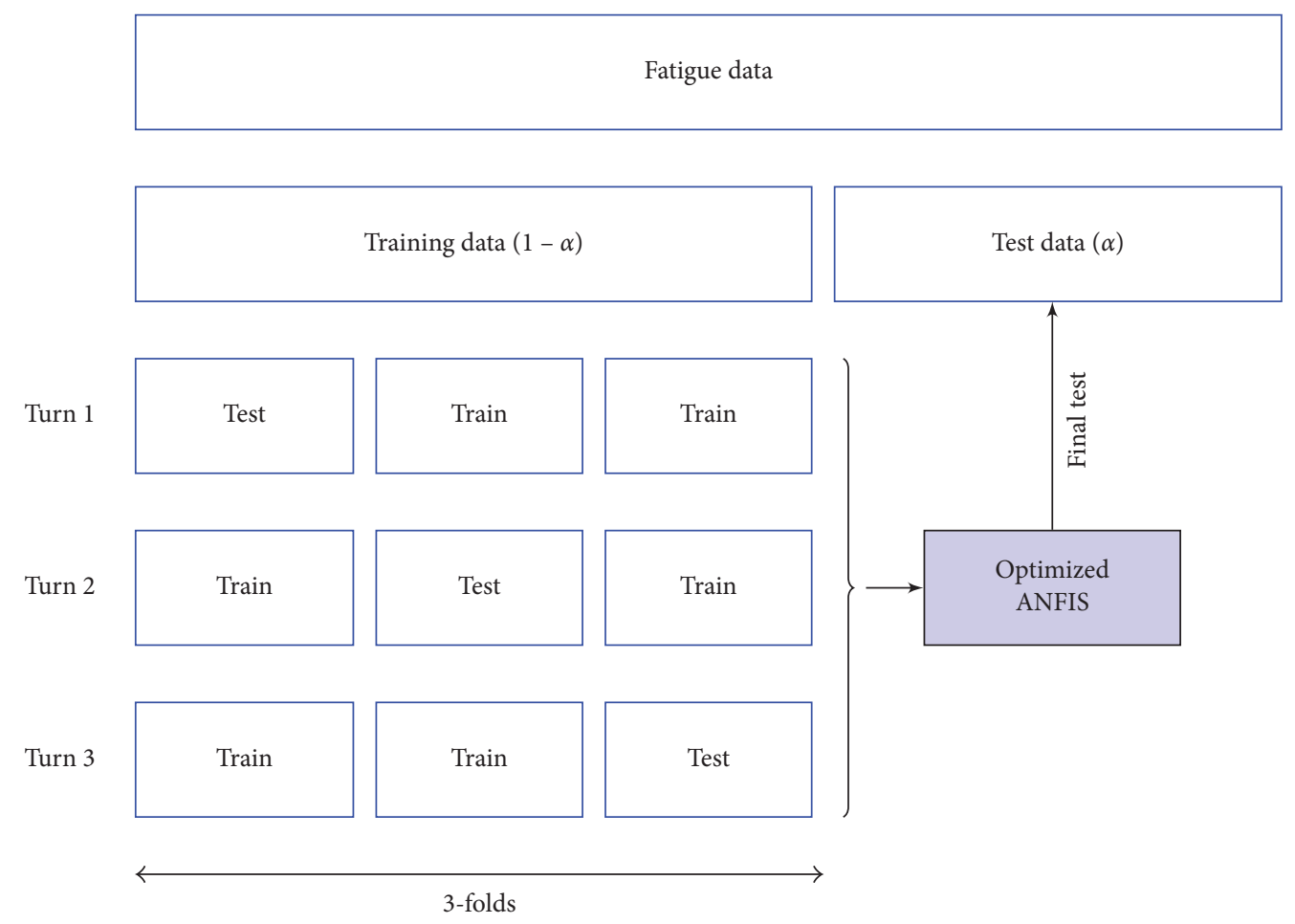

FIGURE 2: Illustration for $k$-fold cross-validation with $k=3$.

\section{The Proposed Method}

This section develops a method for fatigue life prediction of 2-DOF compliant mechanism. The ANFIS model which to be estimated has the form $Y=f\left(X_{1}, X_{2}, \ldots, X_{d}\right)$ where $X_{1}$, $X_{2}, \ldots, X_{d}$ are $d$ design variables and $Y$ is the dependent variable. Using the subtractive clustering technique, the model performance relies on the choice of radius parameters $r_{i}\left(0<=r_{i}<=1, i=1,2, \ldots, d+1\right)$. Therefore, the 10 -fold cross-validation is used and the differential evolution algorithm is utilized for optimizing the mean square error (MSE) average over 10-folds. Because the DE defines a solution by a chromosome, each solution needs to be first encoded. The encoding phase is summarized in Figure 3. As shown in Figure 3, a possible solution is defined as a chromosome. Each chromosome contains $d+1$ genes representing the corresponding radii of the design variables and the dependent variables. The MSE, whose values may be different for different chromosomes, can be considered as an implicit objective function of $r_{i}$.

After the encoding phase is completed, chromosomes or solutions can be handled with mutation, crossover, and selection operators. Through each iteration $i$, only a fixed number of solutions can be selected for the next iteration. After a given number of iterations, maxiter, a chromosome that provides the smallest MSE average over 10 -folds can be considered as the best solution and can be used as the parameters of the ANFIS. The overall process of the proposed method is outlined in Figure 4.

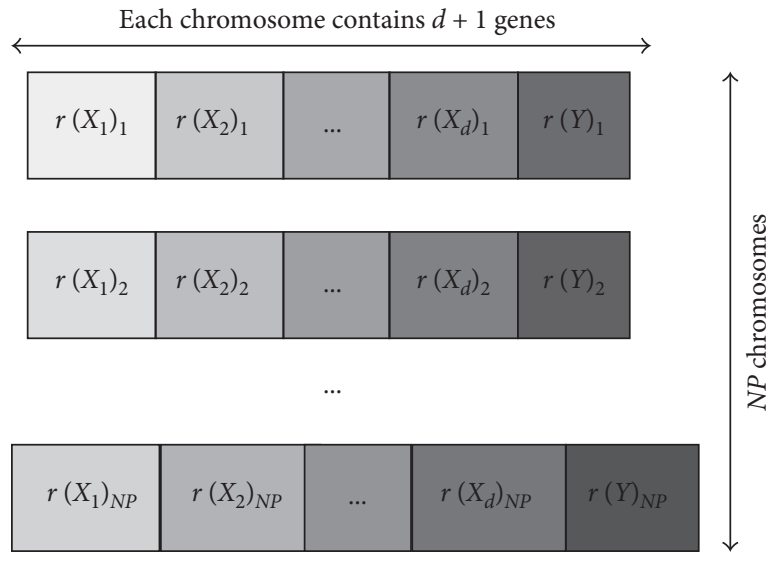

FIGURE 3: Illustration for the encoding phase.

\section{Numerical Example}

4.1. The Data Set. Figure 5 gives a design scheme of a new 2DOF compliant mechanism. This mechanism includes a shuttle table which is located at the middle position. The shuttle table can move in the $x$-direction when a piezostack actuator (PSA-X) applies a force to an end of the table. Besides, when a force comes from the PSA-Y, the shuttle table is moved in the $y$-direction. The overall movement of the shuttle table is based on the elastic deflection of flexure hinges, including flexure hinge 1 (thickness $T_{1}$ and length $V_{1}$ ), flexure hinge 2 (thickness $T_{2}$ and length $\mathrm{H}$ ), and flexure 


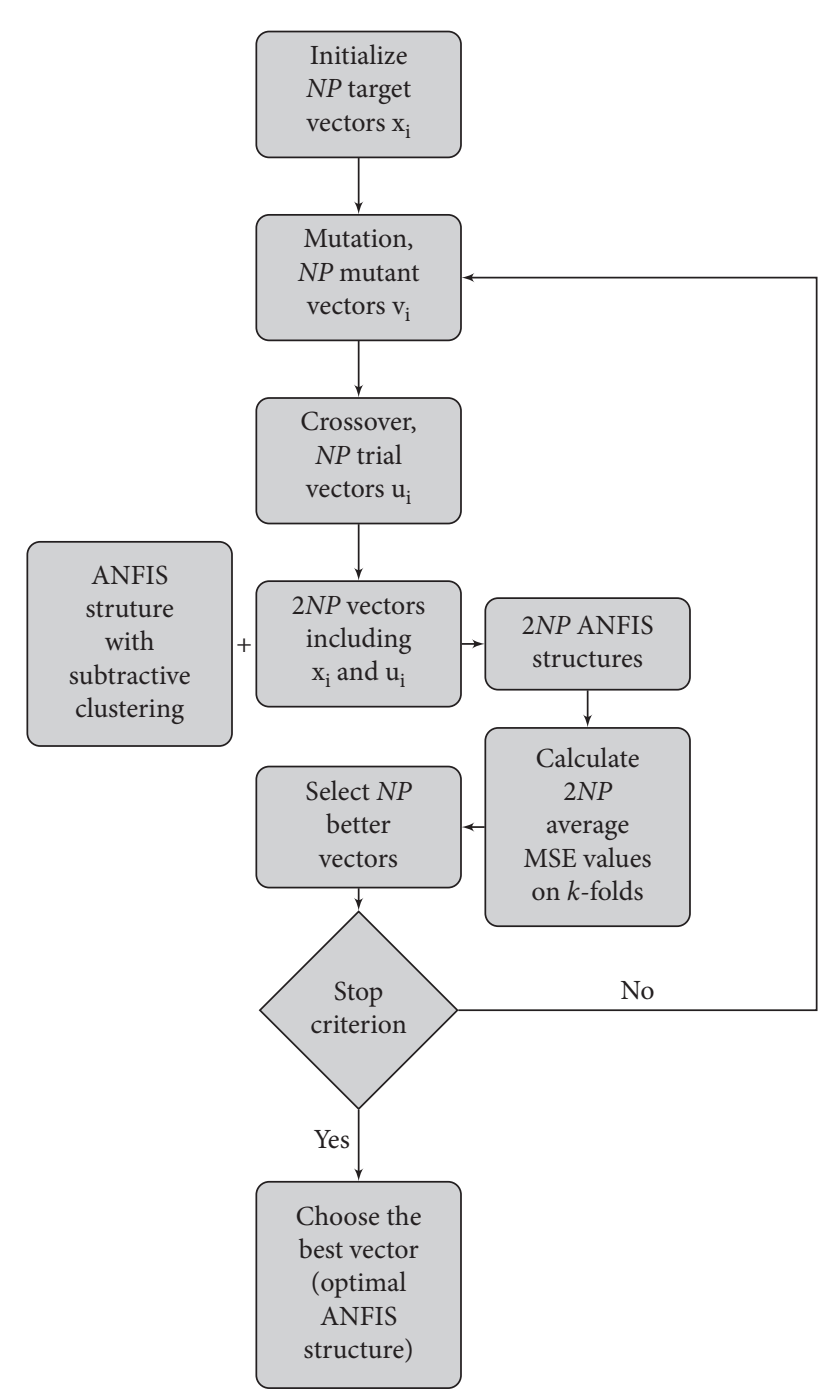

FIgURE 4: The flowchart of the entire method.

hinge 3 (length $V_{2}$ ). Overall dimensions of the mechanism are given, as in Figures 5(a) and 5(b). The width of the mechanism is $b$ of $10 \mathrm{~mm}$ (see Figure 5(c)). It is noted that the motions of flexure hinges are repeated due to these hinges are worked in an elastic area. Therefore, the mechanism is easy to subject a fatigue failure. Predicting fatigue life for the mechanism is critically important to prevent failure and ensure a safety operation.

During the design phase, manufacturing material affects the fatigue life of the 2-DOF mechanism because each material has a specific limitation of strength. This section applies the proposed method for predicting the fatigue life of the new 2-DOF compliant mechanism in two cases: material steel A-36 and material AL 6061-T6. The physical properties, chemical components of steel A-36, and Al 6061-T6 are shown in Table 1, whereas the alternative stresses of the two materials are shown in Table $2[47,48]$. In both cases, a load, $\mathrm{F}$, of $20 \mathrm{~N}$ is applied the shuttle table along the $x$-axis and fixed supports are located by screws at three holes, as depicted in Figure 5.
After boundary conditions are applied for the model, the model is meshed. A 3D model of the 2-DoF mechanism is built for finite element analysis, as shown in Figure 6 . However, uncertainties and errors are critically important for any data. Because the datasets of the fatigue life are determined through finite element simulations in ANSYS software, the numerical datasets are strongly influenced by some controllable parameters. Datasets are therefore also affected by those parameters. First of all, a few main parameters of the meshing model, such as number of elements, number of nodes, and type of elements, are determined to permit a good analysis. Adjusting any parameter also leads to a difference result. So, before implementing the simulations, a meshing quality is evaluated to ensure a good predicting accuracy. The mechanism is meshed with 55376 nodes and 24884 elements. The type of ten-node tetrahedral elements is utilized for the meshing model. Meshing quality is evaluated, as shown in Figure 7. It is noted that a better meshing quality ensures a more simulation accuracy. The results indicated that the meshing distribution is relatively good with a metric average value of 0.2546 and a standard deviation of 0.1891 .

Datasets of the fatigue life are then determined by simulations in ANSYS software R19 and are summarized in Table 3. It can be noted in Table 3 that the range of input variables is set by the user, but the range of output variable is based on the simulated results. Furthermore, the natural $\log$ transformation is applied to the output variable for producing smaller values. The data sets can be also downloaded at https://sites.google.com/tdtu.edu.vn/ nguyentrangthao/home.

\section{Results and Discussion}

The performance of the proposed method is compared (subtractive clustering $+\mathrm{DE}$ ) with four different ANFIS structures consisting of grid partitioning + triangular membership function (Model 1), grid partitioning + bellshaped membership function (Model 2), grid partitioning + Gaussian membership function (Model 3), and subtractive clustering + random range of influence $r$ (Model 4). The considered problems have five design variables and one output variable. For the traditional ANFIS with the grid partitioning method, the number of fuzzy is set as 2 , for each variable. Based on the above information, the parameters of Models 1, 2, and 3 can be determined. Particularly, the number of nodes, the number of linear parameters, and the number of fuzzy rules are, respectively, 92, 192, and 32, for all the three models; the number of nonlinear parameters is 30 for Model 1 and 2 and is 20 for Model 3. For Model 4 and the proposed method, the above parameters do not need to be prior determined but depend upon the value of radius $r$, which are randomly chosen in Model 4 and are optimized using DE in the proposed method. For the proposed method, the convergence behavior depends upon the hyperparameters of $\mathrm{DE}$, such as mutation rate $F$, crossover rate $C R$, and stop criteria. Nevertheless, a comprehensive 


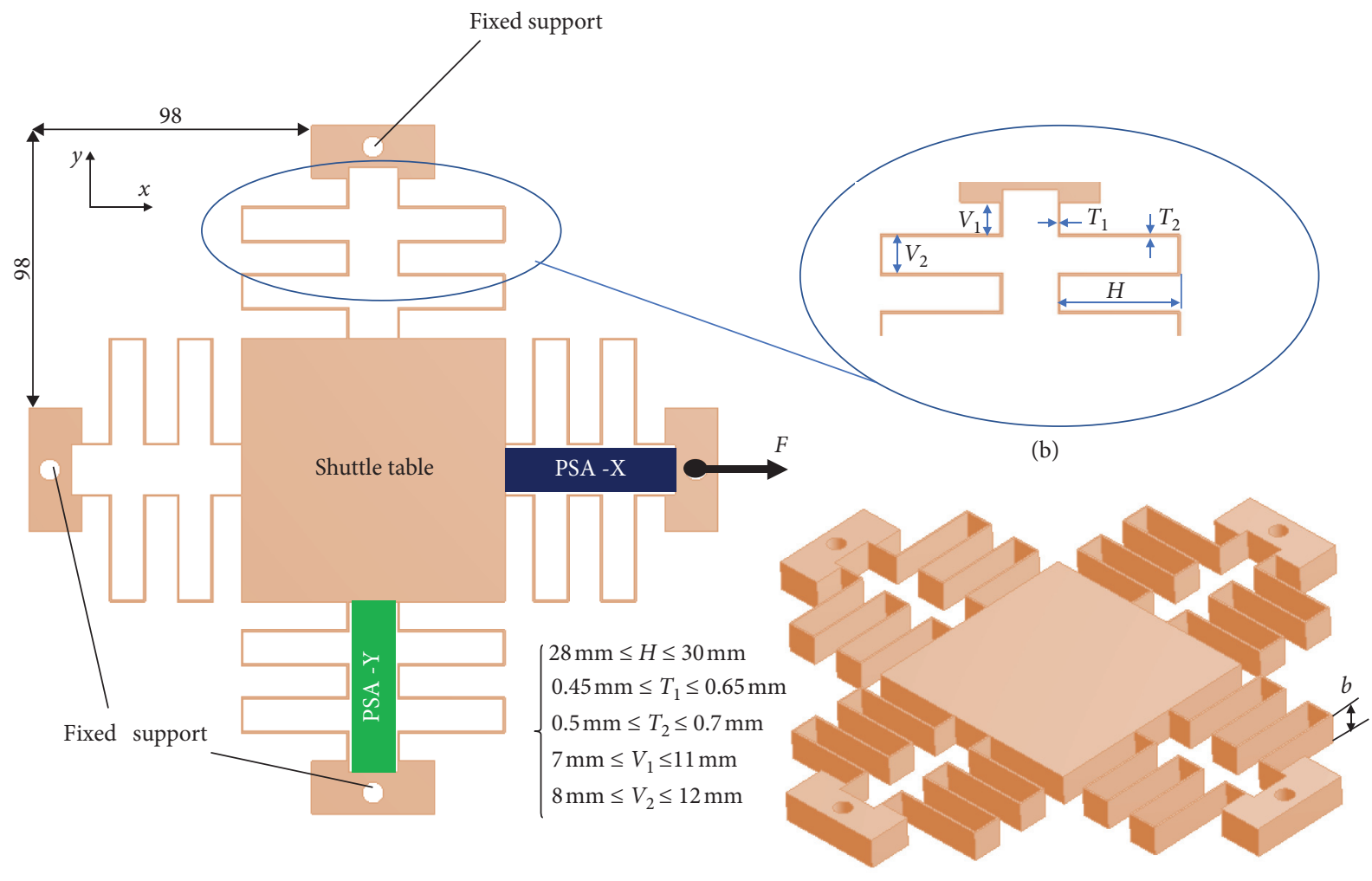

(a)

(c)

Figure 5: 2-DOF compliant mechanism: (a) 2D scheme, (b) dimension, and (c) 3D model (unit: mm).

TABLE 1: Physical properties and chemical components of steel A-36 and Al 6061-T6 (http://asm.matweb.com/search/SpecificMaterial.asp? bassnum=MA6061T6).

\begin{tabular}{lcc}
\hline Physical properties & Steel A-36 & Al 6061-T6 \\
\hline Density $\left(\mathrm{kg} / \mathrm{m}^{3}\right)$ & 7850 & 2700 \\
Young's modulus $(\mathrm{MPa})$ & 200000 & 68900 \\
Poisson's ratio & 0.32 & 0.33 \\
Yield strength $(\mathrm{MPa})$ & 250 & 276 \\
Tensile ultimate strength $(\mathrm{MPa})$ & 550 & 310 \\
Chemical components & (Wt.\%) & (Wt.\%) \\
& Carbon, C: 0.29 & Aluminum, Al: $95.8-98.6$ \\
& Iron, Fe: 98 & Chromium, Cr: $0.04-0.35$ \\
& Copper, Cu: $0.15-0.40$ \\
& Panganese, Mn: $0.8-1.2$ & Iron, Fe: $\leq 0.70$ \\
& Phosphorous, P: 0.04 & Magnesium, Mg: $0.80-1.2$ \\
& Silicon, Si: $0.15-0.4$ & Manganese, Mn: $\leq 0.15$ \\
& Sulfur, S: 0.05 & Silicon, Si: $0.40-0.80$ \\
& & Titanium, Ti: $\leq 0.15$ \\
Zinc, Zn: $\leq 0.25$
\end{tabular}

investigation for all above parameters is inefficient in practice. In this paper, firstly, some hyperparameters of DE are selected based on the investigations in $[43,49,50]$. Specifically, the size of population NP is 20 , the mutation rate $F$ is randomly chosen in the range $[0.4,1]$, crossover rate $C R$ is randomly chosen in the range $[0.7,1]$, and the tolerance $\mathrm{Tol}$ is 10-6. Finally, the number of maximum iterations maxiter is investigated.

Figure 8 illustrates the convergence behavior of DE regarding the iterations, for some cases. It can be seen that the value of objective function sharply decreases at some first iterations. After 10-15 iterations, the value of the objective function still decreases but is not significant. In this study, the number of maximum iteration maxiter is set as 20 to trade off the quality of objective function and the computational cost. In other words, to design an efficient algorithm, a relative optimal solution rather than an exact optimal solution is chosen.

Let $\alpha$ be the ratio of the test set compared to the data set. For each material, the performance of the proposed method for different values of $\alpha$ will be evaluated. For each $\alpha$, the training set is first divided into $k$-folds. Applying the DE 
TABLe 2: The alternative stress for two cases (unit: MPa).

\begin{tabular}{lccc}
\hline & Steel A-36 & & AL 6061-T6 \\
\hline Cycles & Alternative stress & Cycles & Alternative stress \\
10 & 3999 & 1700 & 275.8 \\
20 & 2827 & 5000 & 241.3 \\
50 & 1896 & 34000 & 206.8 \\
100 & 1413 & $1.4 E+5$ & 172.4 \\
200 & 1069 & $8 E+5$ & 137.9 \\
2000 & 441 & $2.4 E+6$ & 117.2 \\
10000 & 262 & $5.5 E+7$ & 89.63 \\
20000 & 214 & $1 E+8$ & 82.74 \\
$1 E+5$ & 138 & & \\
$2 E+5$ & 114 & & \\
$1 E+6$ & 86.2 & & \\
\hline
\end{tabular}

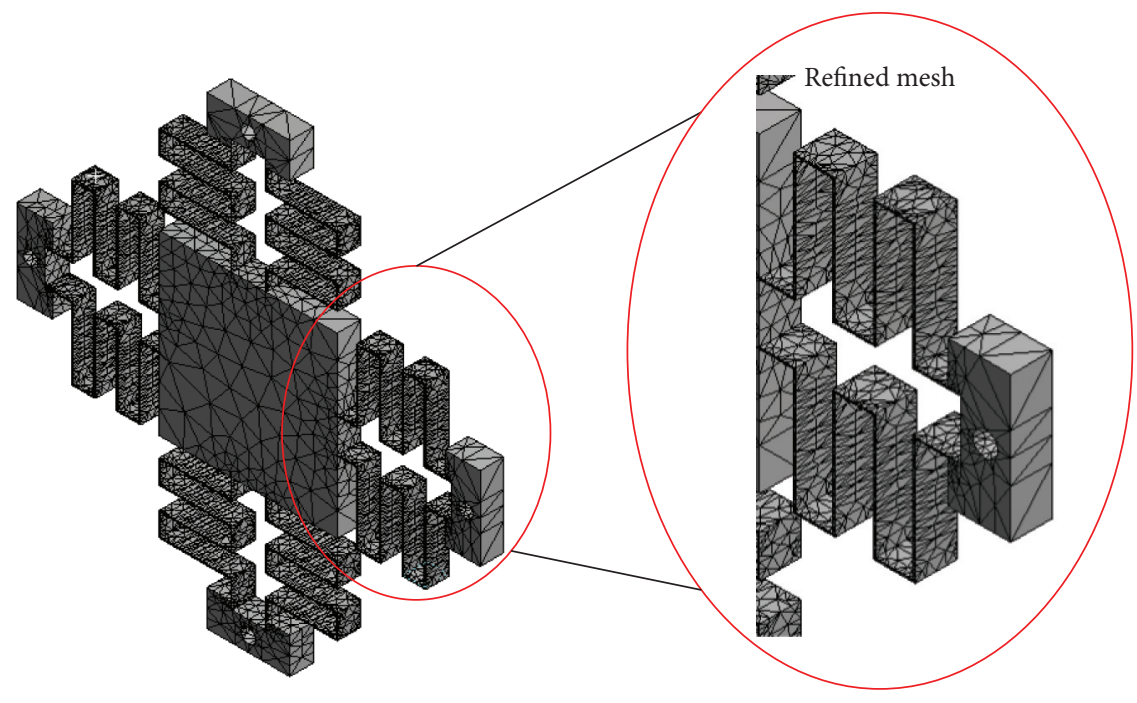

(a)

(b)

Figure 6: Meshed model: (a) mesh for the mechanism; (b) meshing refirement.

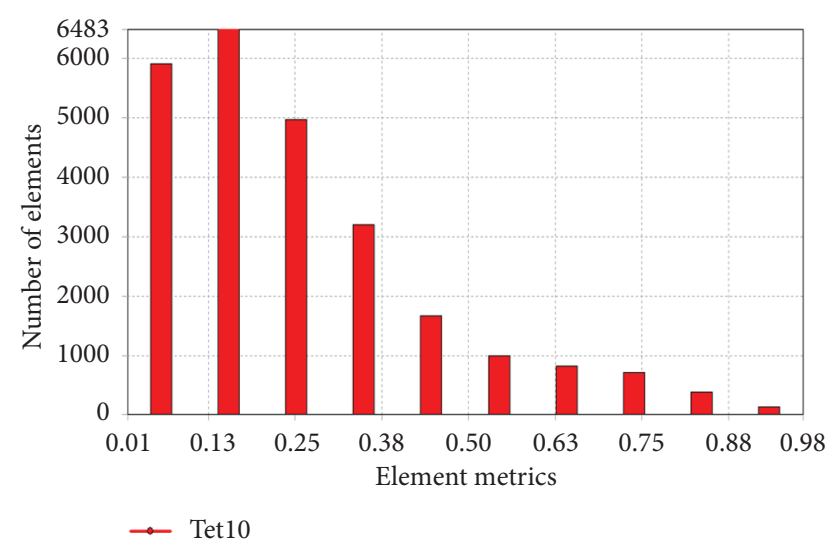

Figure 7: Meshing quality.

algorithm to training data, the optimal ANFIS that minimizes the average MSE (AMSE) over the $k$-folds can be found. Table 4 shows the optimal results for different values of $\alpha$ and materials.
Based on the obtained optimal parameters, the respective ANFIS networks can be built. The obtained models are next evaluated on the test data set. Their predictions are, respectively, shown in Figures 9 and 10, for some typical cases. It can be observed from Figures 9 and 10 that the three ANFIS models with the grid partitioning method result in underfitting in some instances. Meanwhile, most of instances are quite well predicted using the two ANFIS models with the subtractive clustering method. Compared to the subtractive clustering with random values of $r$, the subtractive clustering with DE has a slightly better prediction (9/ 16 and 9/14 samples for Case 1 and Case 2, respectively).

The average MSE of the five comparative ANFIS models for different $\alpha$ values is presented in Figures 11 and 12 (two cases of materials). It can be seen that the average MSE in terms of the natural logarithm of number cycles of the proposed method lies in the range $[0.28,1.92]$ for Case 1 and $[0.50,5.52]$ for Case 2 ; that is, the average RMSEs in terms of the number of cycles of the proposed method lie in the range $[1.7,3.97]$ for Case 1 and $[2.03,10.38]$ for Case 2. These results show that the accuracy of the proposed method is 
TABLE 3: Summary of input/output variables.

\begin{tabular}{lcccccc}
\hline & \multicolumn{3}{c}{ Input } & Output \\
\hline Variable & $T_{1}$ & $T_{2}$ & $V_{1}$ & $V_{2}$ & $H$ & Y: fatigue life \\
\hline Range (unit) & {$[0.45-0.65](\mathrm{mm})$} & {$[0.50-0.70](\mathrm{mm})$} & {$[7-11](\mathrm{mm})$} & {$[8-12](\mathrm{mm})$} & {$[28-30](\mathrm{mm})$} & {$\left[10^{5}-10^{8}\right](\mathrm{cycles})$} \\
\hline
\end{tabular}

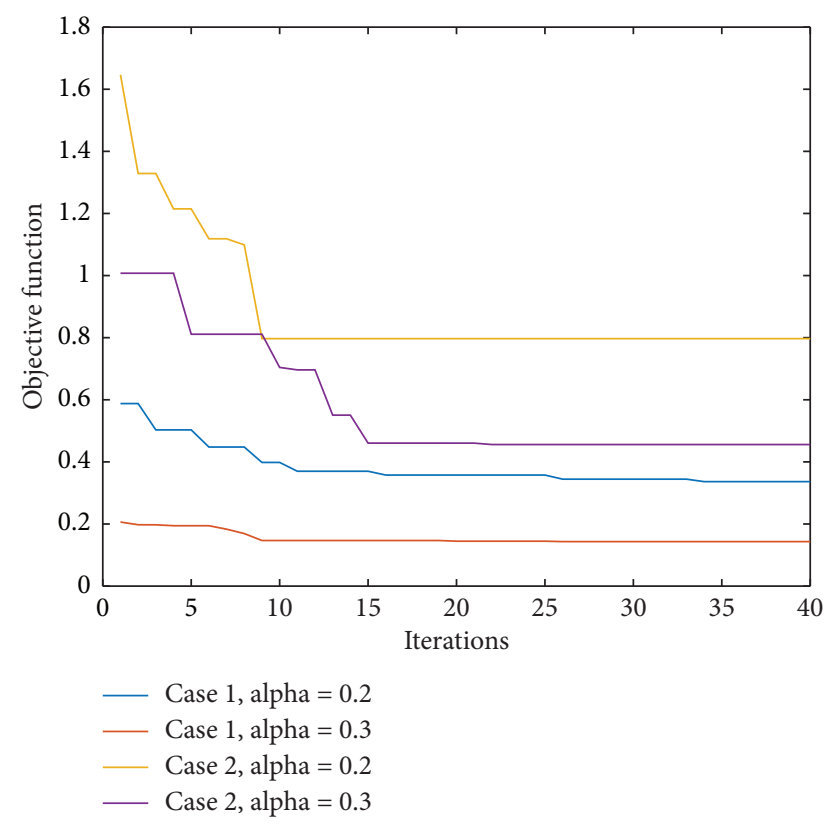

FIgURE 8: The convergence behavior of DE.

TABLE 4: The optimal results for different values of $\alpha$, for each material.

\begin{tabular}{|c|c|c|c|c|c|c|c|}
\hline \multicolumn{8}{|c|}{ Material steel A-36 } \\
\hline$\alpha$ & AMSE & $r_{1}$ & $r_{2}$ & $r_{3}$ & $r_{4}$ & $r_{5}$ & $r_{6}$ \\
\hline 0.1 & 0.2158 & 0.557717 & 0.570578 & 0.900698 & 0.552192 & 0.678305 & 0.949026 \\
\hline 0.2 & 0.3494 & 0.58875 & 0.728122 & 0.988132 & 0.72264 & 0.593982 & 0.842378 \\
\hline 0.3 & 0.1435 & 0.051594 & 0.433607 & 0.322844 & 0.542523 & 0.721756 & 0.991462 \\
\hline 0.4 & 0.1697 & 0.593079 & 0.189186 & 0.571713 & 0.450699 & 0.565966 & 0.838848 \\
\hline 0.5 & 0.3096 & 0.276578 & 0.306819 & 0.651095 & 0.496469 & 0.287918 & 0.370214 \\
\hline 0.6 & 0.1642 & 0.180583 & 0.209091 & 0.985943 & 0.847647 & 0.708588 & 0.939774 \\
\hline \multicolumn{8}{|c|}{ Material Al 6061-T6 } \\
\hline$\alpha$ & AMSE & $r_{1}$ & $r_{2}$ & $r_{3}$ & $r_{4}$ & $r_{5}$ & $r_{6}$ \\
\hline 0.1 & 1.0877 & 0.821216 & 0.305964 & 0.931995 & 0.52941 & 0.906354 & 0.717082 \\
\hline 0.2 & 0.9076 & 0.49391 & 0.636016 & 0.845297 & 0.842738 & 0.990537 & 0.012617 \\
\hline 0.3 & 0.4604 & 0.633621 & 0.286778 & 0.653442 & 0.661226 & 0.882189 & 0.499495 \\
\hline 0.4 & 0.4801 & 0.324963 & 0.534011 & 0.913222 & 0.547666 & 0.951157 & 0.831897 \\
\hline 0.5 & 0.5403 & 0.820838 & 0.415253 & 0.907088 & 0.751343 & 0.781149 & 0.381371 \\
\hline 0.6 & 0.3675 & 0.808686 & 0.06451 & 0.053043 & 0.260961 & 0.576902 & 0.96311 \\
\hline
\end{tabular}

very high. Besides, it can be observed that the effect of the ratio of test data on the performances of two models with subtractive clustering is not so strong. As shown in Figures 11 and 12, in both cases, their average MSEs slightly fluctuate around small values even for high values of $\alpha$. Meanwhile, it can be observed that the average MSEs of other ANFIS models will increase with either too high or too small $\alpha$ values.
The box plots in Figures 13 and 14 provide some statistical visualization of the performance of all methods for two kinds of materials. Among the three ANFIS models using the grid partitioning method, it can be seen from Figures 13 and 14 that the model with triangular membership function has a better result for steel A-36 but not for $\mathrm{Al}$ 6061-T6. Hence, the most suitable membership function cannot be determined. It might depend on which material is 


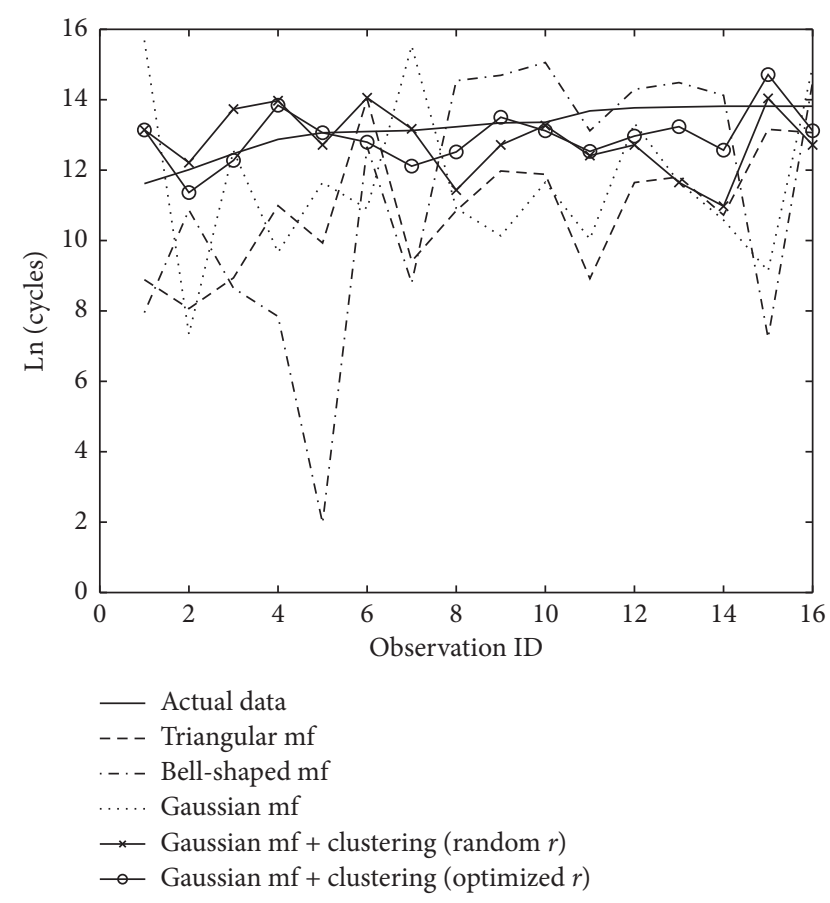

FIGURE 9: The predicted results of comparative methods on the test set (material steel A-36, alpha $=0.6$ ).

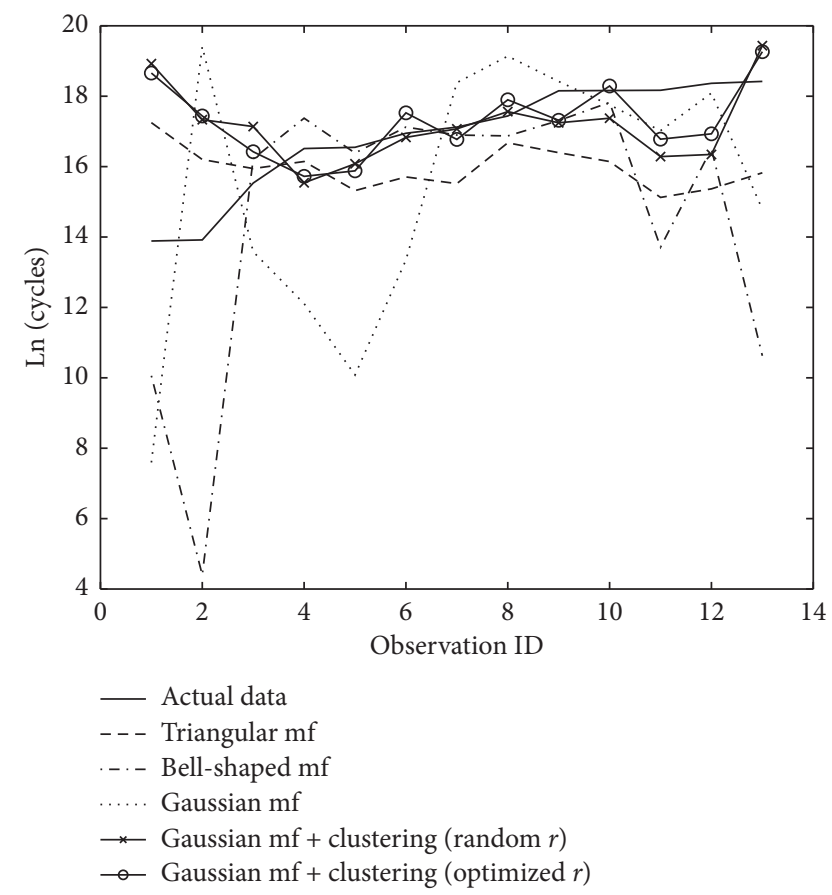

Figure 10: The predicted results of comparative methods on the test set (material Al 6061-T6, alpha $=0.5$ ).

considered. These figures also demonstrate the superiority of the two ANFIS models using subtractive clustering over the other models. In comparison with the subtractive clustering

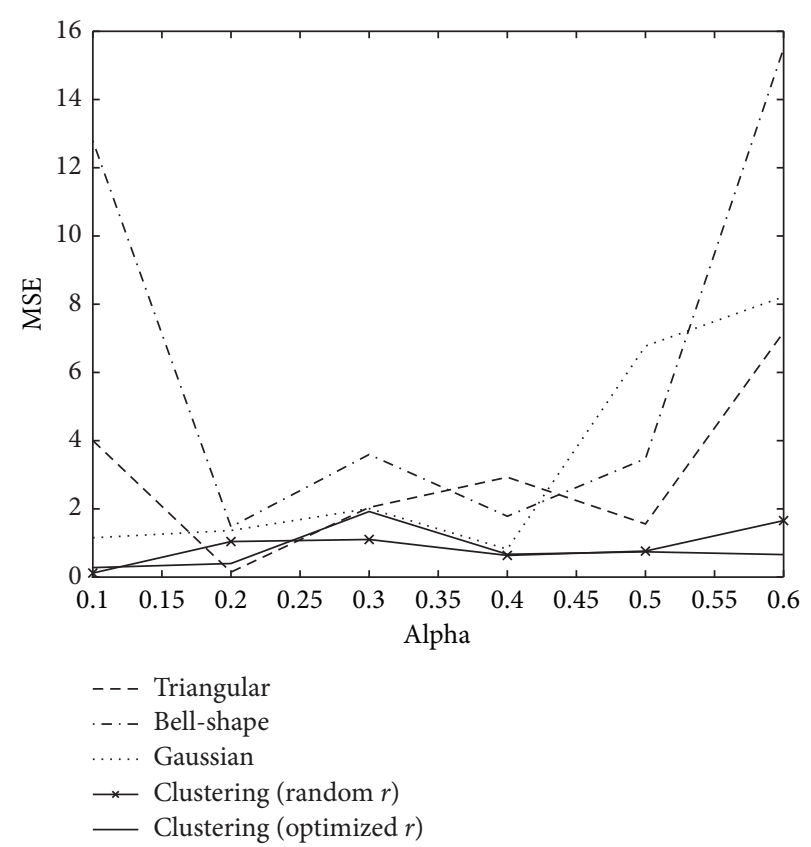

FIGURE 11: MSE of the methods as the ratio of test data increases (steel A-36).

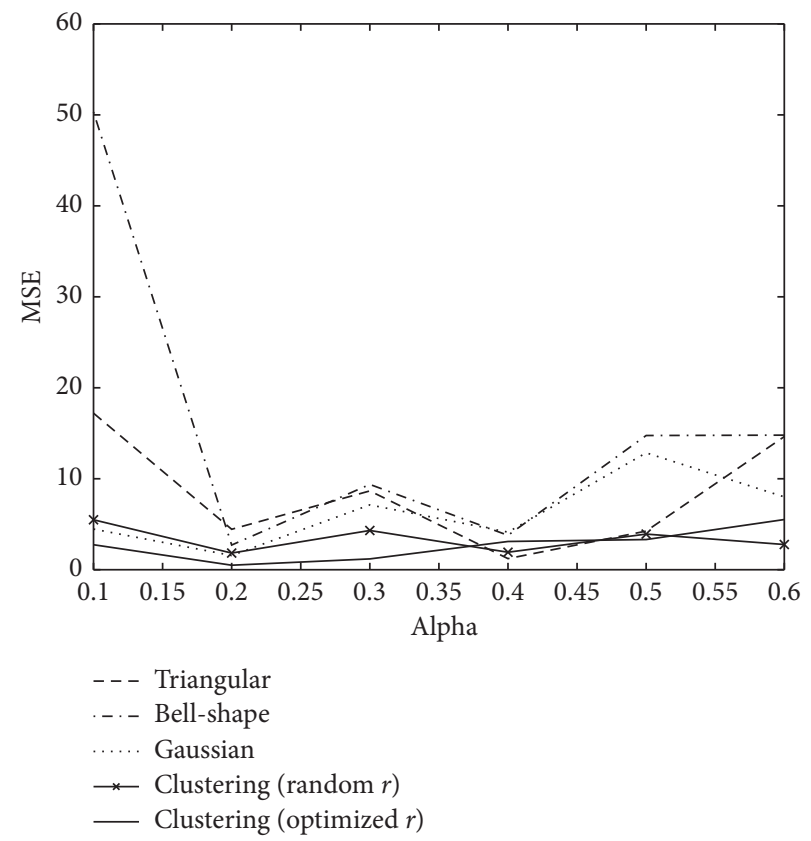

FIGURE 12: MSE of the methods as the ratio of test data increases (Al 6061-T6).

with the random values of $r$, the subtractive clustering with DE has a slightly better performance in both cases. As shown in Figures 13 and 14, the median of the subtractive clustering with DE is lower than those of subtractive clustering with random values of $r$. Besides, the boxes, which stand for the interquartile range (from 25 th percentile to 75 th percentile), 


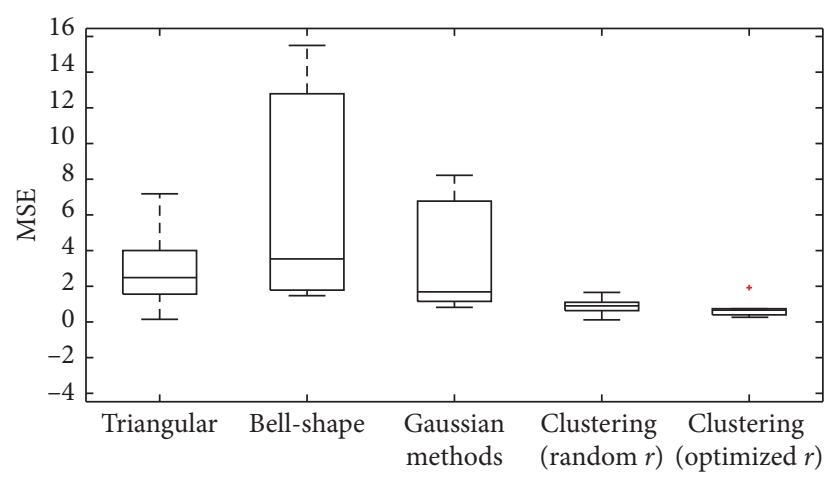

FIgURE 13: Summary of MSE of all methods on test sets (steel A-36).

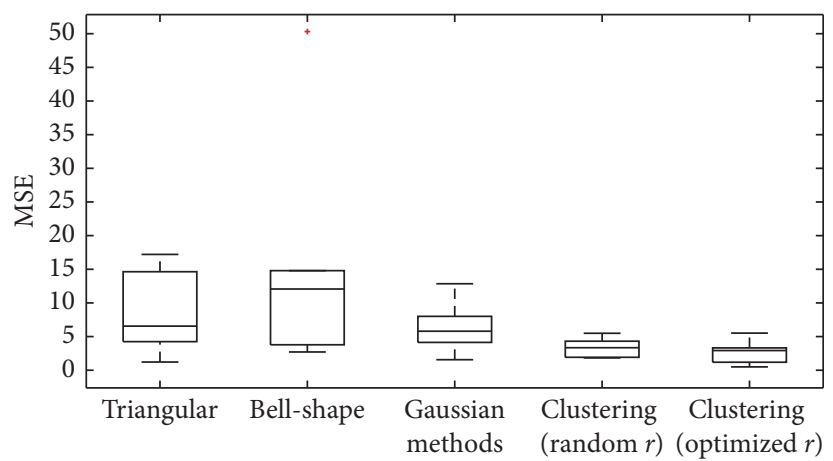

Figure 14: Summary of MSE of all methods on test sets (Al 6061-T6).

of the subtractive clustering with DE are also lower than those of subtractive clustering with random values of $r$, for both cases.

Although Figures 13 and 14 provide the first insight into the performance of all ANFIS models, it is more reliable if a statistical test could be conducted. For this purpose, the Wilcoxon signed-rank test with a statistical significance value of 0.1 is used. The null hypothesis $\mathrm{H} 0$ is built as "there is no difference between the performance of the method $A$ and the performance of the method $B$," where $A$ is the subtractive clustering with $\mathrm{DE}$ and $B$ is a comparative method (grid partitioning + triangular membership function, grid partitioning + bell-shaped membership function, grid partitioning + Gaussian membership function, and subtractive clustering + random range of influence $r$ ). According to Table 5, it can be concluded that the subtractive clustering with DE can improve the performance of ANFIS models using the grid partitioning method, with a significance level of 0.1 . The difference between subtractive clustering with DE and the subtractive clustering with random $r$ is not significant due to the following reasons. Firstly, the difference between the subtractive clustering with $\mathrm{DE}$ and the subtractive clustering with random $r$ is not as large as the differences observed in comparison with other methods. Secondly, the sample size is not large enough for rejection of the null hypothesis $\mathrm{H} 0$. Therefore, the subtractive clustering with random $r$ can be used for other cases in which the computational time is important. On the other hand, more experiments on other ratios of test data $\alpha$ and other materials are required to provide a comprehensive comparison. The results show an efficient application of clustering-based ANFIS in fatigue life prediction of 2-DOF compliant mechanism.

In summary, the above experiments have demonstrated the potential benefit of ANFIS in fatigue life prediction of 2DOF compliant mechanism. Particularly, the two ANFIS models using subtractive clustering provide better performance compared to the three ANFIS models using the grid partitioning method. In comparison with the subtractive clustering with random $r$, the subtractive clustering using DE has slightly better performance, although the difference is not significant. Based on the obtained results, it can be expected that the proposed method can be well-applied for other case studies (materials). The root mean square error of the fatigue life prediction is expected to be smaller than 100 cycles or smaller than 22 in terms of mean square error (MSE) of natural log transformation. Using this criterion, careful users can evaluate the suitability of the proposed method after obtained the training model to decide whether the proposed method can be applied or not. 
TABLE 5: Wilcoxon signed-rank test.

\begin{tabular}{lccc}
\hline & \multicolumn{2}{c}{ Steel A-36 } & \multicolumn{2}{c}{ Al 6061-T6 } \\
& $P$ value & Reject H0 & $P$ value \\
\hline Clustering (optimized r) vs Triangular & 0.09375 & 1 & 0.09375 \\
Clustering (optimized r) vs bell-shape & 0.03125 & 1 & 0.03125 \\
Clustering (optimized r) vs Gaussian & 0.03125 & 1 & 0.03125 \\
Clustering (optimized r) vs clustering (random $r$ ) & 1 & 0 & 0.5625 \\
\hline
\end{tabular}

\section{Conclusions}

This paper for the first time develops a method for fatigue life prediction of 2-DOF compliant mechanism. The adaptive neuro-fuzzy inference system (ANFIS) is utilized for the prediction purpose. In addition to the conventional ANFIS model using the grid partitioning method, the ANFIS model using subtractive clustering is applied. Furthermore, the differential evolution algorithm is utilized for optimizing parameters of the clustering algorithm. Five ANFIS models are applied to two case studies consisting of the steel A-36 and the AL 6061-T6. The obtained results show that the average root mean square error in terms of the number of cycles lies in the range [1.7, 3.97] for Case 1 and $[2.03,10.38]$ for Case 2. Compared to the ANFIS models using the grid partitioning method, the proposed method achieves a better performance with a significance level from 0.05 to 0.1 . Although the subtractive clustering using DE is slightly better than the subtractive clustering with random $r$, the respective difference has no statistical significance. The experiments also demonstrate the potential application of ANFIS in fatigue life prediction of 2-DOF compliant mechanism. The results can be extended in predicting the fatigue life for other compliant mechanisms and related engineering applications. In future work, some physical prototypes can be fabricated to estimate the fatigue life for the proposed 2DOF compliant mechanism.

\section{Data Availability}

The data used to support the findings of this study are available from the corresponding author upon request.

\section{Conflicts of Interest}

The authors declare that they have no conflicts of interest.

\section{Acknowledgments}

This research was funded by the Vietnam National Foundation for Science and Technology Development (NAFOSTED) under grant no. 107.01-2019.14.

\section{References}

[1] T.-P. Dao and S.-C. Huang, "Design and multi-objective optimization for a broad self-amplified 2-DOF monolithic mechanism," Sadhana-Academy Proceedings in Engineering Sciences, vol. 42, pp. 1527-1542, 2017.

[2] W.-L. Zhu, Z. Zhu, Y. Shi, X. Wang, K. Guan, and B.-F. Ju, "Design, modeling, analysis and testing of a novel piezo- actuated XY compliant mechanism for large workspace nanopositioning," Smart Materials and Structures, vol. 25, no. 11, Article ID 115033, 2016.

[3] Q. Xu, "New flexure parallel-kinematic micropositioning system with large workspace," IEEE Transactions on Robotics, vol. 28, no. 2, pp. 478-491, 2012.

[4] T.-P. Dao and S.-C. Huang, "Optimization of a two degrees of freedom compliant mechanism using Taguchi method-based grey relational analysis," Microsystem Technologies, vol. 23, pp. $4815-4830,2017$.

[5] S.-C. Huang and T.-P. Dao, "Multi-objective optimal design of a 2-DOF flexure-based mechanism using hybrid approach of grey-taguchi coupled response surface methodology and entropy measurement," Arabian Journal for Science and Engineering, vol. 41, no. 12, 2016.

[6] S.-C. Huang and T.-P. Dao, "Design and computational optimization of a flexure-based XY positioning platform using FEA-based response surface methodology," International Journal of Precision Engineering and Manufacturing, vol. 17, no. 8, 2016.

[7] B. Ding, Y. Li, X. Xiao, Y. Tang, and B. Li, "Design and analysis of a 3-DOF planar micromanipulation stage with large rotational displacement for micromanipulation system," $M e$ chanical Sciences, vol. 8, no. 1, p. 117, 2017.

[8] B. Ding, Z. Yang, and Y. Li, "Design of flexure-based modular architecture micro-positioning stage," Microsystem Technologies, vol. 26, no. 9, pp. 2893-2901, 2020.

[9] B. Ding, Z.-X. Yang, X. Xiao, and G. Zhang, "Design of reconfigurable planar micro-positioning stages based on function modules," IEEE Access, vol. 7, pp. 15102-15112, 2019.

[10] E. G. Merriam, J. E. Jones, S. P. Magleby, and L. L. Howell, "Monolithic 2 DOF fully compliant space pointing mechanism," Mechanical Sciences, vol. 4, no. 2, pp. 381-390, 2013.

[11] W.-L. Zhu, Z. Zhu, Y. Shi et al., "A novel piezoelectrically actuated 2-DoF compliant micro/nano-positioning stage with multi-level amplification," Review of Scientific Instruments, vol. 87, no. 10, Article ID 105006, 2016.

[12] N. Wang, Z. Zhang, X. Zhang, and C. Cui, "Optimization of a 2-DOF micro-positioning stage using corrugated flexure units," Mechanism and Machine Theory, vol. 121, pp. 683-696, 2018.

[13] M. Ling, J. Cao, Z. Jiang, M. Zeng, and Q. Li, “Optimal design of a piezo-actuated 2-DOF millimeter-range monolithic flexure mechanism with a pseudo-static model," Mechanical Systems and Signal Processing, vol. 115, pp. 120-131, 2019.

[14] J. Yang, G. Kang, Y. Liu, K. Chen, and Q. Kan, "Life prediction for rate-dependent low-cycle fatigue of PA6 polymer considering ratchetting: semi-empirical model and neural network based approach," International Journal of Fatigue, vol. 136, Article ID 105619, 2020.

[15] A. P. Vassilopoulos and R. Bedi, "Adaptive neuro-fuzzy inference system in modelling fatigue life of multidirectional composite laminates," Computational Materials Science, vol. 43, no. 4, pp. 1086-1093, 2008. 
[16] A. P. Vassilopoulos, Fatigue Life Prediction of Composites and Composite Structures, Woodhead Publishing, Sawston, UK, 2019.

[17] C. Anitescu, E. Atroshchenko, N. Alajlan, and T. Rabczuk, "Artificial neural network methods for the solution of second order boundary value problems," Computers, Materials \& Continua, vol. 59, no. 1, pp. 345-359, 2019.

[18] E. Samaniego, C. Anitescu, S. Goswami et al., "An energy approach to the solution of partial differential equations in computational mechanics via machine learning: concepts, implementation and applications," Computer Methods in Applied Mechanics and Engineering, vol. 362, Article ID 112790, 2020.

[19] Q. Liu, W. Shi, and Z. Chen, "Rubber fatigue life prediction using a random forest method and nonlinear cumulative fatigue damage model," Journal of Applied Polymer Science, vol. 137 , no. 14 , p. $48519,2020$.

[20] J. C. Stinville, W. C. Lenthe, M. P. Echlin, P. G. Callahan, D. Texier, and T. M. Pollock, "Microstructural statistics for fatigue crack initiation in polycrystalline nickel-base superalloys," International Journal of Fracture, vol. 208, no. 1-2, pp. 221-240, 2017.

[21] K. V. Anderson and S. R. Daniewicz, "Statistical analysis of the influence of defects on fatigue life using a Gumbel distribution," International Journal of Fatigue, vol. 112, pp. 78-83, 2018.

[22] T. Loutas, N. Eleftheroglou, and D. Zarouchas, "A data-driven probabilistic framework towards the in-situ prognostics of fatigue life of composites based on acoustic emission data," Composite Structures, vol. 161, pp. 522-529, 2017.

[23] D. Shi and H. Ma, "A bayesian inference method and its application in fatigue crack life prediction," IEEE Access, vol. 7, pp. 118381-118387, 2019.

[24] W. Sai, G. B. Chai, and N. Srikanth, "Fatigue life prediction of GLARE composites using regression tree ensemble-based machine learning model," Advanced Theory and Simulations, vol. 3, no. 6, Article ID 2000048, 2020.

[25] D. Nowell and P. W. Nowell, "A machine learning approach to the prediction of fretting fatigue life," Tribology International, vol. 141, Article ID 105913, 2020.

[26] M. Zhang, C.-N. Sun, X. Zhang et al., "High cycle fatigue life prediction of laser additive manufactured stainless steel: a machine learning approach," International Journal of Fatigue, vol. 128, Article ID 105194, 2019.

[27] Y. Al-Assaf and H. El Kadi, "Fatigue life prediction of unidirectional glass fiber/epoxy composite laminae using neural networks," Composite Structures, vol. 53, no. 1, pp. 65-71, 2001.

[28] J. A. Lee, D. P. Almond, and B. Harris, "The use of neural networks for the prediction of fatigue lives of composite materials," Composites Part A: Applied Science and Manufacturing, vol. 30, no. 10, pp. 1159-1169, 1999.

[29] Z. La, "Fuzzy sets," Information and Control, vol. 8, pp. 338-353, 1965.

[30] T. Takagi and M. Sugeno, "Fuzzy identification of systems and its applications to modeling and control," IEEE Transactions on Systems, Man, and Cybernetics, vol. 15, no. 1, pp. 116-132, 1985.

[31] J.-S. R. Jang, “ANFIS: adaptive-network-based fuzzy inference system," IEEE Transactions on Systems, Man, and Cybernetics, vol. 23, no. 3, pp. 665-685, 1993.

[32] D. Dinh-Cong, S. Pham-Duy, and T. Nguyen-Thoi, "Damage detection of $2 \mathrm{D}$ frame structures using incomplete measurements by optimization procedure and model reduction,"
Journal of Advanced Engineering and Computation, vol. 2, no. 3, pp. 164-173, 2018.

[33] H. Naderpour and M. Mirrashid, "Shear failure capacity prediction of concrete beam-column joints in terms of ANFIS and GMDH," Practice Periodical on Structural Design and Construction, vol. 24, no. 2, Article ID 4019006, 2019.

[34] H. Naderpour and M. Mirrashid, "Moment capacity estimation of spirally reinforced concrete columns using ANFIS," Complex \& Intelligent Systems, vol. 6, no. 1, pp. 97-107, 2020.

[35] R. Mikaeil, S. S. Haghshenas, Y. Ozcelik, and H. H. Gharehgheshlagh, "Performance evaluation of adaptive neuro-fuzzy inference system and group method of data handling-type neural network for estimating wear rate of diamond wire saw," Geotechnical and Geological Engineering, vol. 36, no. 6, pp. 3779-3791, 2018.

[36] A. R. Dormishi, M. Ataei, R. Khaloo Kakaie, R. Mikaeil, and S. Shaffiee Haghshenas, "Performance evaluation of gang saw using hybrid ANFIS-DE and hybrid ANFIS-PSO algorithms," International Journal of Surface Mining Reclamation and Environment, vol. 10, no. 2, pp. 543-557, 2019.

[37] A. Aryafar, R. Mikaeil, S. S. Haghshenas, and S. S. Haghshenas, "Application of metaheuristic algorithms to optimal clustering of sawing machine vibration," Measurement, vol. 124, pp. 20-31, 2018.

[38] Y. Huang, Y. Lan, S. J. Thomson, A. Fang, W. C. Hoffmann, and R. E. Lacey, "Development of soft computing and applications in agricultural and biological engineering," Computers and Electronics in Agriculture, vol. 71, no. 2, pp. 107-127, 2010.

[39] R. Babuška, Fuzzy Modeling for Control, vol. 12, Springer Science \& Business Media, Berlin, Germany, 2012.

[40] T. VoVan and T. NguyenTrang, "Similar coefficient for cluster of probability density functions," Communications in Statistics-Theory and Methods, vol. 47, no. 8, pp. 1792-1811, 2018.

[41] S. L. Chiu, "Fuzzy model identification based on cluster estimation," Journal of Intelligent and Fuzzy Systems, vol. 2, no. 3, pp. 267-278, 1994.

[42] R. Storn and K. Price, "Differential evolution-a simple and efficient heuristic for global optimization over continuous spaces," Journal of Global Optimization, vol. 11, no. 4, pp. 341-359, 1997.

[43] N.-T. Thao, "An improved fuzzy time series forecasting model using the differential evolution algorithm," Journal of Intelligent \& Fuzzy Systems, vol. 36, no. 2, pp. 1727-1741, 2019.

[44] G. J. McLachlan, K.-A. Do, and C. Ambroise, Analyzing Microarray Gene Expression Data, vol. 422, John Wiley \& Sons, Hoboken, NJ, USA, 2005.

[45] J. Zhou, X. Li, and H. S. Mitri, "Classification of rockburst in underground projects: comparison of ten supervised learning methods," Journal of Computing in Civil Engineering, vol. 30, no. 5, Article ID 4016003, 2016.

[46] G. Vanwinckelen and H. Blockeel, "On estimating model accuracy with repeated cross-validation," in Benelearn 2012: Proceedings of the 21st Belgian-Dutch Conference on Machine Learning, pp. 39-44, Ghent, Belgium, March 2012.

[47] T. L. Boyer and E. Howard, Metals Handbook, American Society for Metals, Materials Park, OH, USA, 1985.

[48] P. D. Harvey, Engineering Properties of Steel, ASM International, Cleveland, OH, USA, 1982.

[49] V. Ho-Huu, T. Nguyen-Thoi, T. Vo-Duy, and T. NguyenTrang, "An adaptive elitist differential evolution for optimization of truss structures with discrete design variables," Computers \& Structures, vol. 165, pp. 59-75, 2016. 
[50] T. Nguyen-Trang, T. Nguyen-Thoi, T. Truong-Khac, A. T. Pham-Chau, and H. Ao, "An efficient hybrid optimization approach using adaptive elitist differential evolution and spherical quadratic steepest descent and its application for clustering," Scientific Programming, vol. 2019, Article ID 7151574, 2019. 